\title{
Influence of Synchronized Dead Point Elimination Crank on Cyclist Muscle Fatigue
}

\author{
Khadijah Akmal Abdul Aziz ${ }^{1,}$, Ahmad Faizal S ${ }^{1}$., Norasmadi Abdul Rahim ${ }^{1}$, Mohd. \\ Hanafi Mat Som ${ }^{1}$, and Hamzah Sakeran ${ }^{1}$ \\ ${ }^{1}$ School of Mechatronic Engineering, University Malaysia Perlis, Perlis, Malaysia
}

\begin{abstract}
The aim of this study was to investigate the influence of newly proposed bicycle's crank to crank angle setting on the Vastus Lateralis (VL) and Bicep Femoris (BF) muscle activity during cycling. Procedures of Conconi Test were used throughout the experiment for the data collection purpose. The muscles activities were recorded using surface electromyography and software LabChart7. The raw data were further processed in time (Root-Mean-Square, RMS) and frequency (Mean Power Frequency, MPF) domain. It was found that $0^{\circ}$ crank to crank setting (similar to conventional crank to crank angle setting) caused the prime mover VL (Normalized RMS $=0.119$ ) to fatigue more than BF (Normalized RMS $=0.102$ ). This setting is expected to decrease the cycling performance. In addition, $-5^{\circ}$ is the best crank to crank angle setting that causes least fatigue to both $\mathrm{VL}$ and $\mathrm{BF}$. In short, to increase the cycling performance by avoiding the fatigue to the main muscles, $-5^{\circ}$ is the suggested as setting angle for the proposed crank design.
\end{abstract}

\section{Introduction}

Cycling is one of the most famous sport worldwide, which is suitable for all ages and providing a lot of benefits such as good for health and also for the environment. One of the main components that make the bicycles move is the crank which can be manipulated to improve the cycling performance especially during competition. Several strategies to optimize the cycling power are the alteration of the crank arm length, chairing geometry and position of the chainring rotation axis [1]. Generally, the conventional cranks are separated at $180^{\circ}$ and were reported to produce minimal power when one of the legs reaches Bottom Dead Centre (BDC) while the other leg is at Top Dead Centre (TDC) [2]. Those two points so called dead points because throughout the circular motion of cycling, TDC and BDC produce minimum tangential force [3]. Previous research found that by avoiding both leg to reach TDC and BDC simultaneously, the net cycling power will be increased and thus enhance the cyclist performance [4]. Thus, the proposed crank is able to prevent the legs reaching TDC and BDC simultaneously. In this study, the performance of the cyclists was measured based on the level of cyclist's muscle fatigue. This can be achieved by analyzing the muscles activity in time and frequency domain which were

*Corresponding author: ahmadfaizal@unimap.edu.my 
recorded using surface electromyography. Therefore, in this research, the influence of the proposed crank on the cyclist muscles fatigue is investigated.

\section{Methodology}

\subsection{Subjects}

This study involved 10 normal and healthy subjects, which consisted of 5 males and 5 females (age $23.10 \pm 0.32$ years; weight $57.1 \pm 12.84 \mathrm{~kg}$; height $1.63 \pm 0.12 \mathrm{~m}$; Body Mass Index (BMI) $21.26 \pm 2.52 \mathrm{~kg} / \mathrm{m}^{2}$ ). The subjects were healthy without any muscular, neurological and tendineous injuries. Each subject read and signed the written informed consent form. The details about the study and the procedures were presented to them clearly before the experiments were conducted.

\subsection{Experimental Equipment}

The brand of the bicycle used in this study is Polygon Helios 100. An interactive smart trainer with an electrobrake system, Tacx Vortex Smart T2180 was attached to the rear tire of the bicycle to provide suitable training resistance to the cyclist. The proposed crank is shown in Figure 1.
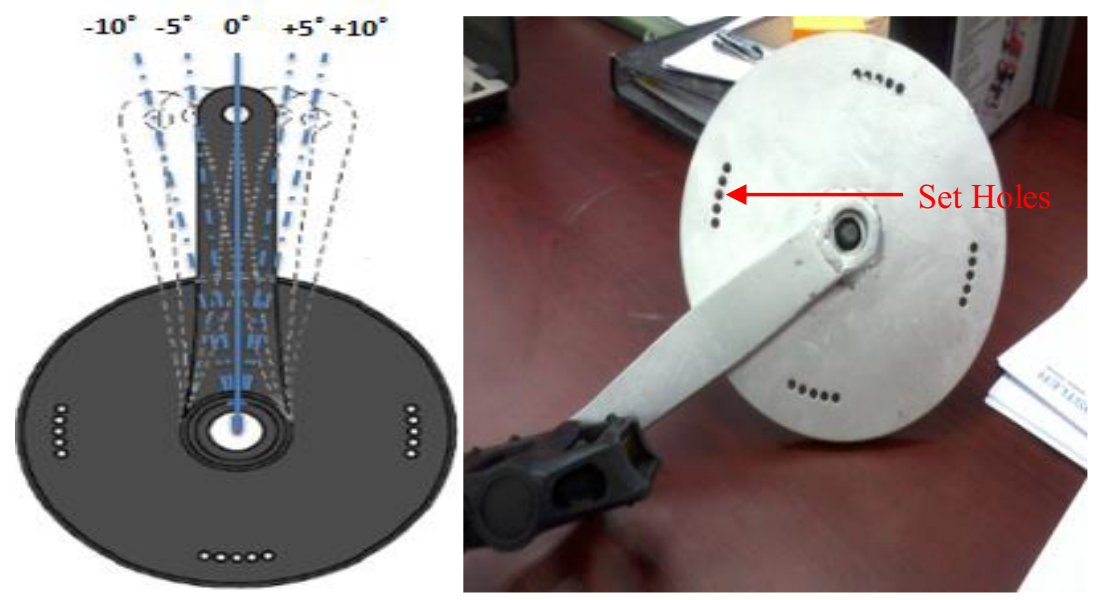

Fig. 1. Proposed Crank System.

The Polygon Helios 100 bicycle installed with the proposed crank on the left side of the bicycle. The proposed crank has two pieces of circle shape disc where one disc (no crank attaches to the disc) is fixed to the bottom bracket of the bicycle and the other (crank was welded to the disc) is freely rotated and could be fixed by screwing through few 'set holes' to the first disc for a desired crank angular position. The crank able to be set for $\pm 10^{\circ}$ maximum with the addition of $5^{\circ}$ back and forth from inertial $0^{\circ}$ of TDC point. The $0^{\circ}$ resembles the conventional crank which the left and right cranks are separated at $180^{\circ}$. An EMG (ML865, ADInstrument) was used to monitor muscles activity. EMG signals were amplified and recorded at a sampling rate of $2000 \mathrm{~Hz}$. 


\subsection{Experimental Protocol}

The electrode placement on the quadriceps (VL) and hamstring (BF) of the subjects are according to the Surface ElectroMyoGraphy for the Non-Invasive Assessment of Muscles (SENIAM) recommendations. The subjects followed the Conconi Test throughout the cycling activity. The subjects started cycling after a warmed-up of 5 minutes with a workload of $70 \mathrm{~W}$. Subjects kept increasing the speed until no longer able to continue the cycling experiment (fatigue). Breathing difficulties or 'burning muscles' are the examples of physical indications of near-maximal power [10]. During cycling protocol, the subject need to increase both cadence and force. This will resemble the actual cycling conditions when cyclists reach their maximal speed at a cadence exceeding 160 revolutions per minute [11].

\subsection{Data Analysis}

MATLAB R2013a was opted to filter the raw data using Fourth order Butterworth band pass filter $(20 \mathrm{~Hz}-450 \mathrm{~Hz})$. The peak value represents the dynamic maximum voluntary contraction (MVC). For normalization of the data, the filtered data were divided by the peak value. Then, Root-Mean-Square (RMS) method is used for the muscle fatigue analysis. The RMS is in the time domain. Next, the data is further analysed in the frequency domain. Then, Mean Power Frequency (MPF) which is the centroid frequency of the power spectrum being used in this study [12].

\section{Result and discussion}

Firstly, the activity of BF and VL were investigated individually to understand the effect of varying crank to crank angles on the activity of those muscles. Besides, this analysis is crucial to examine the relationship of those muscles and to decide the best angle to avoid the severe muscle fatigue.

In Fig. 2, $0^{\circ}$ crank to crank angle (similar to conventional crank) shows the least amount of RMS value for both VL and BF. However, only at $0^{\circ}$ and $-10^{\circ}$, the RMS value of VL is higher than $\mathrm{BF}$. For the other angles $\left(+10^{\circ},+5^{\circ},-5^{\circ}\right)$, the RMS value of $\mathrm{BF}$ is higher than VL. Generally, from these two observations, $0^{\circ}$ and $-10^{\circ}$ cause the prime mover muscle, VL fatigue more. This occurrence should be highly avoided because prime mover is responsible for the specific movement and antagonist muscle helps the prime mover to accomplish the movement. 


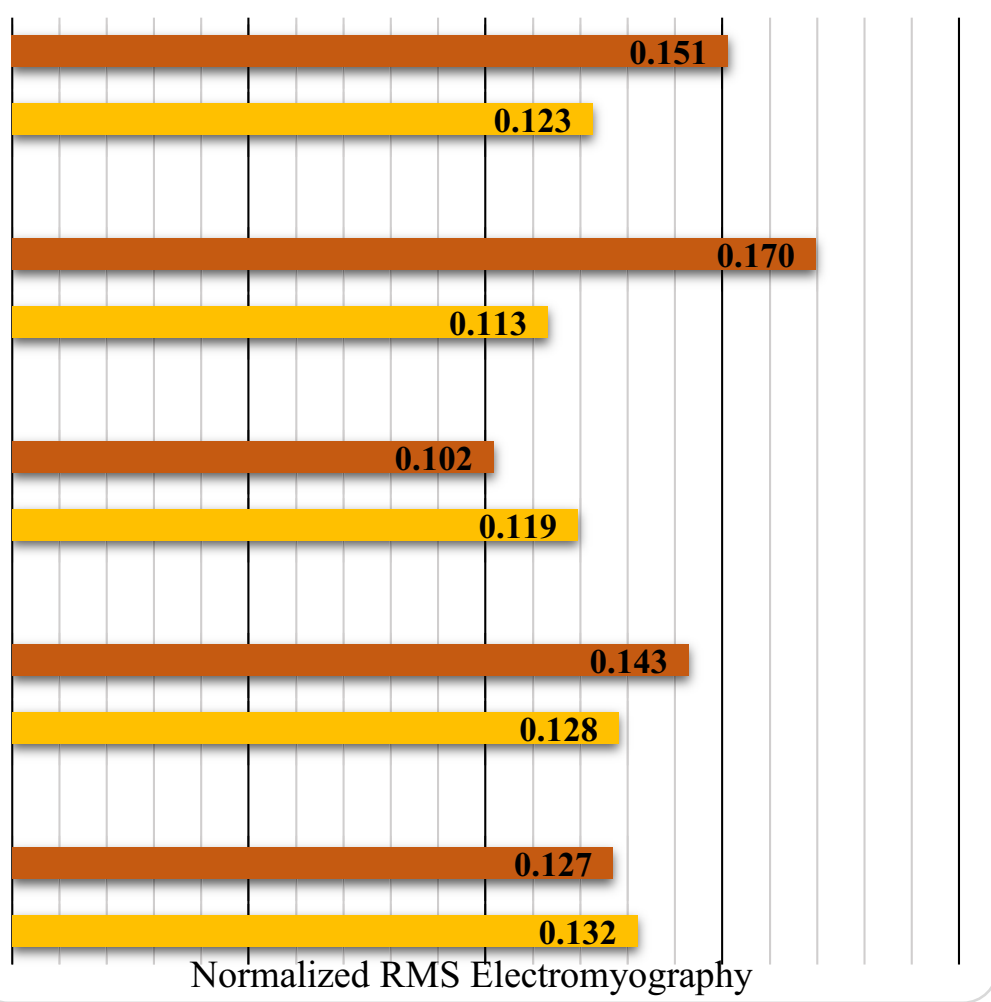

Fig. 2. RMS of VL and BF for 5 different crank angle.

In addition, the antagonist muscle, $\mathrm{BF}$ consumes much more energy at $+10^{\circ},+5^{\circ}$ and $-5^{\circ}$ during the pulling phase. In Fig. 2, it is shown that at least only one muscle was affected as the crank to crank angles being varied. This gives a good impact to the cyclist because the proposed crank design did not severely affect both antagonistic-pair muscles simultaneously, which could lead to the failure of cycling activity in both propulsive and pulling phase. In short, $\mathrm{BF}$ shows higher RMS value at $+10^{\circ},+5^{\circ}$ and $-5^{\circ}$. However, at $0^{\circ}$ and $-10^{\circ}$, VL is having higher RMS value.

Next, the analysis of EMG signal in time domain on the effect of muscle fatigue to both males and females was conducted. Overall, females show the high RMS value for both muscles and for the proposed crank to crank angles as shown in both Fig. 3 and Fig. 4. Females have the higher rate of fatigue levels compared to the males. Apparently, the lifestyle of the females differs from the males. The males quite fit and used to exercise regularly compared to females. 


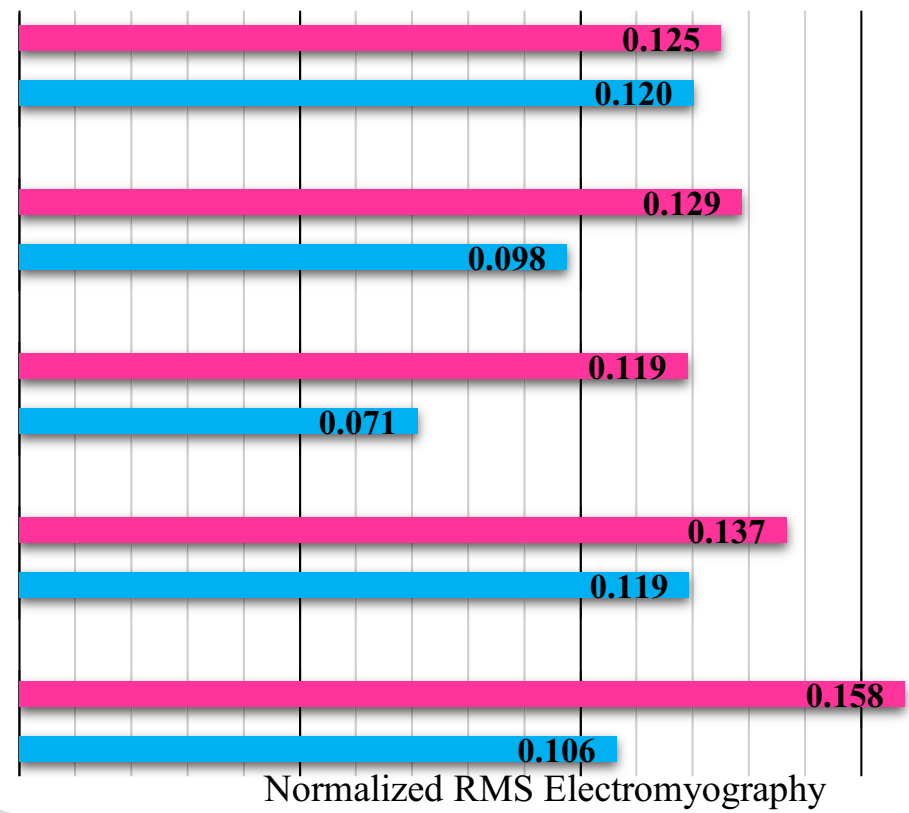

Fig. 3. RMS value of VL activity for male and female.

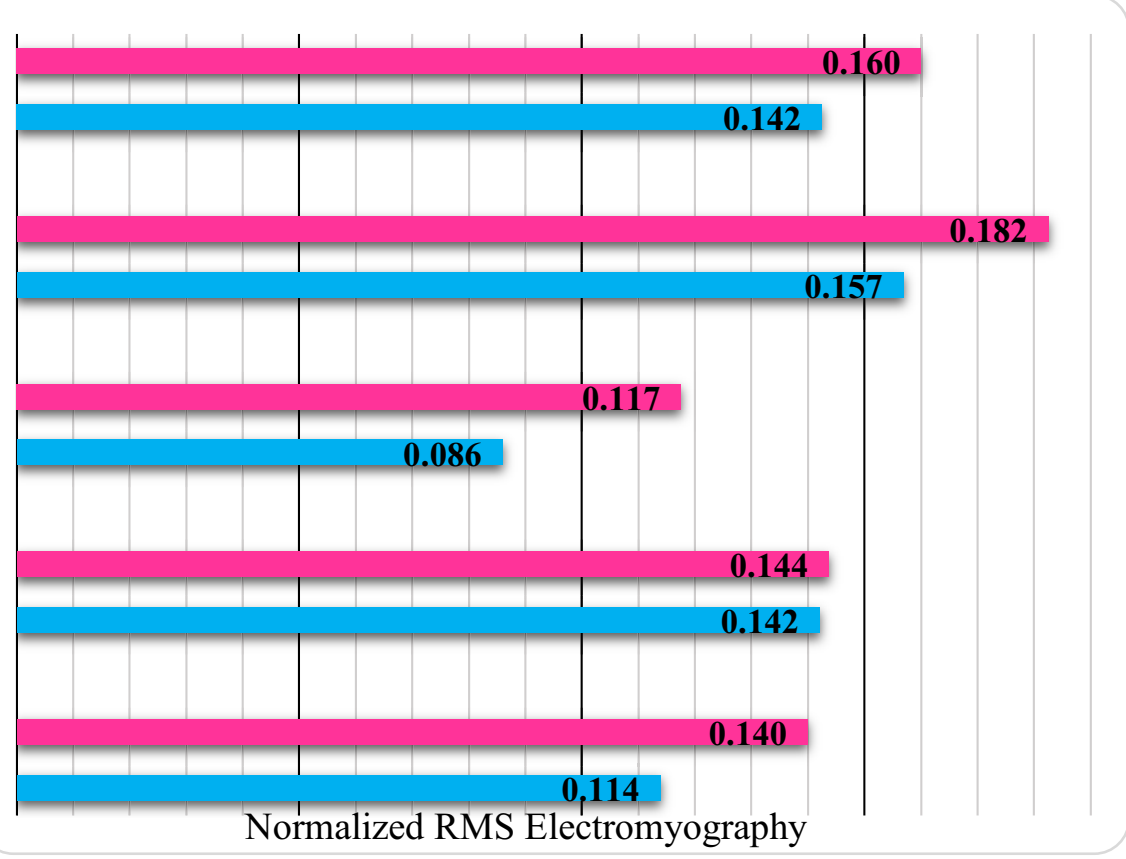

Fig. 4. RMS value of BF activity for male and female. 


\section{Conclusion}

From the analysis, the best crank to crank angle settings that causes least fatigue to both VL and $\mathrm{BF}$ of the subject and does not severely affect the prime mover muscle is $-5^{\circ}$. Conversely, $0^{\circ}$ and $-10^{\circ}$ are the worst angles because they cause fatigue to the prime mover or so called as agonist muscle (VL). Generally, females tend to get fatigued easier in every each experiment. Male able to cycle in a longer period of time compared to the female.

\section{References}

1. R.C.H. So, J.K.F. Ng, G.Y.F. Ng, Phys. Ther. Sport, 6(2), 89 (2005)

2. V. Parnabas, T. Wahidah, N.M. Abdullah, Proceedings of the International Colloquium on Sports Science, Exercise, Engineering and Technology, Singapore, 2014

3. A. Gefen, M. Megido-ravid, Y. Itzchak, M. Arcan, Gait \& Posture, 15, 56 (2002)

4. A. Lucia, J. Balmer, R.C.R. Davison, M. Perez, A. Santalla, P. M. Smith, Int. Journal of Sports Medicine 25(7), 479 (2004)

5. F.M. Giovanni Grazzi, Ilario Casoni, Gianni Mazzoni, Int. Journal of Sports Medicine 17(7), 509 (1996)

6. A. Ignjatovi, P. Hofmann, D. Radovanovi, Physical Education and Sport, 6, 1 (2008)

7. S.R. Alty, A. Georgakis, Signal Processing Conference, 1387 (2011)

8. I. Stirn, T. Jarm, Vojko Strojnik, Kinesiologia Slovenica, 14(1), 28 (2008)

9. C. Fritschi, L. Quinn, J. Psychosom. Res., 69(1), 33 (2010)

10. A. Saito, K. Watanabe, H. Akima, Hum. Mov. Sci. 40, 14 (2015)

11. D.M. Rouffet, C.A. Hautier, J. Electromyogr. Kinesiol., 18, 866 (2008)

12. L. Fedorowich, K. Emery, B. Gervasi, J.N. Cote, J. Electromyogr. Kinesiol., 23(5), $1183(2013)$

13. W.J. Albert, A.T. Wrigley, R.B. McLean, G.G. Sleivert, Dynamic Medicine, 5(2), 1 (2006)

14. A. Phinyomark, S. Thongpanja, H. Hu, INTECH, Croatia, 2012 